\title{
Relationship Between Laser Doppler Signals and Anatomy During Deep Brain Stimulation Electrode Implantation Toward the Ventral Intermediate Nucleus and Subthalamic Nucleus
}

\author{
Karin Wårdell, Peter Zsigmond, Johan Richter and Simone Hemm
}

\section{Linköping University Post Print}

\section{Tweet}

N.B.: When citing this work, cite the original article.

Original Publication:

Karin Wårdell, Peter Zsigmond, Johan Richter and Simone Hemm, Relationship Between Laser Doppler Signals and Anatomy During Deep Brain Stimulation Electrode Implantation Toward the Ventral Intermediate Nucleus and Subthalamic Nucleus, 2013, Neurosurgery, (72), 2, 127-140.

http://dx.doi.org/10.1227/NEU.0b013e31827e5821

Copyright: Lippincott, Williams \& Wilkins http://www.lww.com/

Postprint available at: Linköping University Electronic Press http://urn.kb.se/resolve?urn=urn:nbn:se:liu:diva-90470 


\section{Relation between laser Doppler signals and anatomy during deep brain stimulation electrode implantation towards Vim and STN}

Karin Wårdell, $\mathrm{MSc}, \mathrm{PhD}^{1}$, Peter Zsigmond, $\mathrm{MD}^{2,3}$, Johan Richter, $\mathrm{MD}^{1,2}$, Simone Hemm $\mathrm{MSc}, \mathrm{PhD}^{1,4}$

${ }^{1}$ Department of Biomedical Engineering, Linköping University, Linköping, Sweden

${ }^{2}$ Department of Neurosurgery, Linköping University Hospital, Linköping, Sweden

${ }^{3}$ Department of Clinical and Experimental Medicine, Linköping University, Linköping,

Sweden

${ }^{4}$ University of Applied Sciences Northwestern Switzerland, Institute for Medical and Analytical Technologies, Muttenz, Switzerland

\section{Corresponding author:}

Karin Wårdell

Department of Biomedical Engineering

Linköping University

58185 Linköping, Sweden

Email: karin.wardell@liu.se

Phone: +46-10-1032455

Financial support: The study was supported by a Group Grant (No. 311-2006-7661) from the Swedish Governmental Agency for Innovation Systems (Vinnova), the Swedish Foundation for Strategic Research (SSF), the Swedish Research Council (VR) and by NovaMedTech (funded by the European Union and the Swedish Agency for Economic Growth) as well as from Swiss National Science Foundation (SNF).

Acknowledgement: The authors would like to express their gratitude to Research Engineer Per Sveider, Department of Biomedical Engineering, Linköping University for skillful construction of the mechanical device, and to the Staff at the Department of Neurosurgery, Linköping University Hospital for assistance during the clinical measurements. The support with the Surgiplan ${ }^{\circledR}$ features from Johan Falk, Elekta Instruments $A B$ is also highly appreciated. 


\section{Abstract}

BACKGROUND: Deep brain stimulation (DBS) requires precise and safe navigation to the chosen target. Optical measurements allow for monitoring of gray-white tissue boundaries (total light intensity, TLI) and microvascular blood flow during stereotactic procedures.

5 OBJECTIVE: To establish the link between TLI/blood flow and anatomy along trajectories towards the ventral intermediate nucleus (Vim) and subthalamic nucleus (STN).

METHODS: Stereotactic laser Doppler measurements were done with mm-precision from cortex towards Vim (n=13) and STN (n=9). Optical trajectories of TLI and blood flow were created and compared with anatomy by superimposing the Schaltenbrandt-Wahren atlas on the patients' pre-and postoperative images. Measurements were divided into anatomical subgroups and compared statistically.

RESULTS: Typical TLI-trajectories with well-defined anatomical regions could be identified for Vim and STN. TLI was significantly lower $(\mathrm{P}<.001)$ and microvascular blood flow significantly higher $(\mathrm{P}=.01)$ in the Vim targets. Out of 1285 sites, 38 showed blood flow peaks, 27 of them along Vim trajectories. High blood flow was more common close to sulci and in the vicinity to $\mathrm{Cd} / \mathrm{Put}$. Along one Vim trajectory a small bleeding was suspected during insertion of the probe and confirmed with post-operative CT.

CONCLUSIONS: Laser Doppler is useful for intra-operative guidance during DBS implantation as simultaneous measurement of tissue grayness and microvascular blood flow can be done along the trajectory with mm-precision. Typical but different TLI-trajectories were found for Vim and STN.

Keywords: deep brain stimulation (DBS), laser Doppler perfusion monitoring (LDPM), microvascular blood flow, optical navigation, brain atlas

Running title: Laser Doppler for intra-operative DBS-guidance 


\section{Introduction}

Intracerebral interventions such as deep brain stimulation (DBS) ${ }^{1,2}$ radio-frequency lesioning ${ }^{3}$ or neuronal cell grafting ${ }^{4}$ require accurate, precise and safe navigation to the chosen target ${ }^{5}$. In some groups, supplementary techniques such as impedance measurements ${ }^{6,7}$ or microelectrode recording (MER) ${ }^{8}$ are used to get additional information about the target area preoperatively identified on MR and CT images. Recently, optical measurements have been introduced as emerging technology which is a method for real-time presentation of grey-white tissue boundaries during stereotactic procedures. A thin probe with dimensions adapted to the stereotactic system and with optical fibers aligned along the interior side of the probe towards the tip is connected to the optical system. The probe is used to create tracks for the DBS electrodes along the precalculated trajectories. Up to date Giller et al have performed reflectance spectroscopy recordings in the near infrared region and have shown a high correlation with microelectrode recording and imaging data 9,10 . The concept developed by Wårdell and colleagues, which is based on both laser Doppler perfusion monitoring (LDPM) 11 and diffuse reflection spectroscopy ${ }^{12}$, has been clinically evaluated and compared to impedance recordings ${ }^{13}$. This comparison showed a relation between the signals, but that optical measurements presented a better differentiation between white and grey matter of different shades. Furthermore, it was shown that the microvascular perfusion i.e. blood flow could be recorded along the trajectory at fixed sites.

Up to now, signal differences between grey and white matter have been identified ${ }^{11,13}$. Giller already tried to correlate the anatomical position of each measurement site with the obtained spectroscopy signal ${ }^{10}$. No correlation with the anatomical position has been performed so far for the total light intensity (TLI) representing tissue grayness, and microvascular blood flow due to either the small number of measurement sites or due to continuous recording without any link to the exact anatomic position. In consequence, the aim of the present study was to establish the link between anatomy and TLI as well as microvascular blood flow recordings by means of a mechanical device allowing to push down the optical probe in defined steps from the cortex down to the target, and to correlate the recordings with the different brain structures passed through. 


\section{Material and Methods}

\section{Patients}

Twelve patients (one women, age $44-77$, mean \pm s.d. $=67 \pm 11$ ) referred for unilateral or bilateral DBS-implantation for the treatment of essential tremor or Parkinson's disease were included in the study. In total 22 leads were implanted. Thirteen were implanted in the ventral intermediate nucleus (Vim) and nine in the subthalamic nucleus (STN) respectively. One of the STN-patients had bilateral RF-lesions in Vim. The surgical procedures were done with local anaesthesia for the Vim and general anaesthesia for the STN patients. The study was approved by the local ethics committee at the University Hospital in Linköping (D. no. M18204, T54-09) and informed written consent was received from the patients.

\section{Laser Doppler system and measurement probes}

The system for intracerebral recordings of microvascular blood flow and TLI comprises a laser Doppler perfusion monitor (PF 5010, Perimed AB, Sweden), a specially designed optical probe, mechanical device and a personal computer with software for acquisition, data analysis and presentation. A schematic overview of the system is presented in Fig. 1a and a photo documentation of the probe as positioned with the mechanical device in the Leksell ${ }^{\circledR}$ Stereotactic System (Elekta Instrument AB, Sweden) in Fig 1b.

The measurement probe was constructed in order to fit in the Leksell ${ }^{\circledR}$ Stereotactic System (LSS, Model G, Elekta Instrument AB, Sweden) ${ }^{11,13}$. The probe's outer shaft is rigid, made of stainless steel and has a functional length of $190 \mathrm{~mm}$. The diameter of the probe is $2.2 \mathrm{~mm}$ except for the last $30 \mathrm{~mm}$ towards the tip where it is $1.5 \mathrm{~mm}$ in diameter. Four optical fibers $\left(\varnothing_{\text {core }}=125 \mu \mathrm{m}, \varnothing_{\text {core+cladding }}=140 \mu \mathrm{m}, \varnothing_{\text {total }}=250 \mu \mathrm{m}\right.$, numerical aperture $\left.=0.37\right)$ are aligned along the interior side of the probe towards the tip. Two of the fibers are used for laser Doppler recordings and the two additional fibers can be used for e.g. reflectance spectroscopy registration. In order to minimize tissue trauma during probe insertion but still keep the optical fibers intact, the probe tip is smoothly rounded and the fiber tips carefully polished. To keep the measurement equipment on a safe distance from the operation area, an optical cable length of at least $4 \mathrm{~m}$ is recommended. With the used construction the probe and cable were possible to sterilize according to the STERRAD ${ }^{\circledR}$ protocol ${ }^{14}$.

With the described probe design optical signals were recorded in a small sampling volume in front of the probe. One of the fibers delivers laser light $(1 \mathrm{~mW}, \lambda=780 \mathrm{~nm})$ towards the tissue 
and after interaction with the moving red blood cells and static tissue, a fraction of the light is backscattered to a receiving fiber connected to the detector unit. After low-pass and band-pass filtering of the differentiated electric current from the photo detector, two signals are extracted: the TLI corresponding to the DC-part of the signal and the stochastic fluctuating photo detector signal which is further processed to the perfusion signal ${ }^{15}$. The software, developed in Labview (National Instruments Inc., USA), made it possible to sample, store and present both the perfusion and TLI signals in real-time as well as to perform post-processing and analysis of captured signals ${ }^{16}$. The total range of the perfusion and TLI signals are presented as 0 - 999 arbitrary units (a.u.) and $0-10.0$ a.u. respectively. A TLI > 0.5 is required in order to process the perfusion i.e. blood flow signal by the laser Doppler system.

During intracerebral measurements, the TLI signal should represent the tissue's reflectivity which is related to tissue type (gray matter reflects less resulting in lower TLI than white matter) whereas the perfusion is related to relative changes in the tissue's microcirculation. Perfusion and TLI levels were therefore checked in Motility Standard (Perimed AB, Sweden), a calibration solution, before and after the measurement sessions in order to ascertain comparable levels. When necessary the system was recalibrated. During recordings the sampling time in the software was set to $\mathrm{f}_{\mathrm{s}}=100 \mathrm{~Hz}$ and the time constant $\tau=0.03$.

\section{Mechanical guidance device}

A mechanical device allowing step-wise insertion of the probe from cortex towards the target area was constructed (Fig. 1b). The mechanical device was designed to fit the stereotactic system and made it possible to insert the probe with sub millimeter-precision by moving a hand controlled screw easily visible from above by the surgeon. The hand controlled screw movement gave rise to a pronounced artifact in the optical signals. With this design a total trajectory length of $80 \mathrm{~mm}$ is available.

The accuracy and precision of the mechanical device was investigated experimentally. The mechanical device was fixated to the LSS and the probe was moved in $1 \mathrm{~mm}$ steps from the starting point to the center of the LSS. For every $10^{\text {th }}$ complete turn of the screw the distance to target was controlled. This was repeated three times. The probe reached the target all three times. Furthermore measurements were done in the Motility Standard solution at the same time as the probe was moved down by the mechanical device. In order to keep the probe in the solution, this was only done for approximately $5 \mathrm{~mm}$. This experiment confirmed the peak 
appearing during the turn of the screw and that the perfusion signal caused by the Brownian motion in the Motility immediately reappears when the mechanical device stopped moving.

\section{Surgical technique and measurement procedure}

The surgical procedure followed the general protocol for routine DBS-implantation at the clinic. Stereotactic CT imaging (slice thickness $1 \mathrm{~mm}$, GE Lightspeed Ultra, GE Healthcare, UK) was performed after placement of the LSS. The Schaltenbrandt atlas coordinates for the Vim nucleus were used; 5-7 $\mathrm{mm}$ anterior to the posterior commissure, at the level of the intercommissural line, and $13-15 \mathrm{~mm}$ lateral to the midline of the $3^{\text {rd }}$ ventricle. Direct anatomical targeting of the STN was done on a 1.5 Tesla MRI (T1 and T2, slice thickness 2 $\mathrm{mm}$, Philips Intera, The Netherlands). The trajectories to the target point in the STN were calculated by image fusion of the preoperative MRI scan and CT scan by using Leksell Surgiplan $^{\circledR}$ (Elekta Instrument AB, Sweden). At surgery, the probe was inserted according to the pre-calculated trajectory by use of the mechanical device. If ring or arc angles were adjusted the new values were noted.

All measurement sequences were presented on-line in the operating theatre both for the surgeon and the engineer performing the measurements. During the introduction of the probe, optical measurements of both the TLI and microvascular blood flow were performed every $\mathrm{mm}$ starting in the cortex $55-65 \mathrm{~mm}$ from the pre-calculated target and going down towards the target area. Each mm step was notified by the surgeon and also seen as a movement artifact peak in the blood flow signal. The time for the appearing peak was noted by the engineer responsible for the measurement. Each recording between peaks lasted 5-10 s. The total measurement sequence took approximately 15 minutes per trajectory. When reaching the target area, the optical probe position was verified intra-operatively with fluoroscopy (Philips BV Pulsera, Philips Medical Systems, The Netherlands). When in the target the optical probe was removed and replaced by the DBS electrode $3389^{\circledR}$ (Medtronic Corporation, Minneapolis, MN, USA), whose position was verified by fluoroscopy. The clinical results on awake patients were evaluated with intra-operative test stimulation. As a last step, the extension cable and the pulse generator were implanted in general anesthesia during the same surgical session. The entire procedure including frame positioning, stereotactic imaging, planning, surgical preparation, optical measurements, electrode and pulse generator implantation as well as test stimulation if awake patients took 6-7 hrs. A postoperative CT scan was performed $(n=10)$ in order to confirm the electrode position and used trajectory. In 
total TLI and blood flow was recorded at 616 and 669 measurement positions for Vim and STN respectively. This resulted in a total of 2570 measurement values corresponding to 1285 TLI and microvascular blood flow values respectively.

\section{Data analysis}

\section{Calculation of optical trajectories}

All measurement sequences were visually inspected and compared to the noted time and the corresponding movement artifact created by the mechanical device. The movement artifacts were used as reference for identification of the different measurement sites along the trajectory during the analysis. For each measurement site, after removing the artifact, TLI and blood flow were averaged and the corresponding standard deviation was determined by the Labview software, together with the heart rate. Based on the analyzed values, optical trajectories were defined and plotted for the TLI and the microvascular blood flow. An example of a cut out of the on-line raw signal as presented in the operating room is shown in Fig. 2a and the corresponding calculated TLI and blood flow trajectories after removal of movement artifacts in Fig. $2 b$.

\section{Identification of anatomy}

The postoperative CT was co-registered with the preoperative images and the SchaltenbrandtWahren atlas was superimposed using the Surgiplan ${ }^{\circledR}$ software. If the ring or arc angles had been changed during surgery, new trajectories were added and used instead for the comparison. The final electrode trajectories were then identified on the postoperative CT images based on the electrode artifact ${ }^{17}$ and projected on the preoperative planning image in order to analyze the positions of the different measurements without the disturbing electrode artifact. In the two patients for whom postoperative CT images were not available, the planned trajectory coordinates were used for analysis. Furthermore, planned and final trajectories were compared. By using the oblique mode, together with presentations in axial, coronal and sagital directions, the trajectory was followed $\mathrm{mm}$ by $\mathrm{mm}$ from the target area towards the cortex. The anatomical structures passed were noted for all three directions and used together for identification of anatomical areas. Furthermore, the closest distance to the ventricle $\left(\mathrm{d}_{\mathrm{v}}\right)$ and the distance $\left(\mathrm{l}_{\mathrm{v}}\right)$ to the target from this point were measured.

The Surgiplan ${ }^{\circledR}$ analysis was used to relate regions and structures in the optical trajectories to the anatomy. The following measures were used: the length of the section(s) passed in the internal capsule IC $\left(\mathrm{L}_{\mathrm{IC}}\right)$; the length of the passage through the bridge between cauduate 
nucleus Cd and Putamen (Put) ( $\mathrm{L}_{\mathrm{CdPut}}$ ), the distance from the border between the IC and the thalamus to the Vim-target $\left(\mathrm{L}_{\mathrm{Th}}\right)$. In Figure 3a-b relevant regions in the Schaltenbrandt atlas coronal planes $+2 \mathrm{~mm}$ and $+5 \mathrm{~mm}$ are marked for Vim and STN respectively.

\section{Comparison between TLI-trajectories and anatomy}

The TLI-trajectories were divided into two groups: Vim and STN. In order to make interpatient comparisons between groups, the values for each TLI trajectory were normalized to the average value of the eight highest consecutive values recorded in white matter. For Vim and STN patients measurements were divided into anatomical subgroups following the identified structures: I) cortex, II) white matter (IC), III) the bridge between Cd/Put causing a dip in the TLI-signal or to the vicinity of ventricle (Cd/Put/Ventricle), and IV) target area (including $-4 \mathrm{~mm}$ before the target down to the target).

Wilcoxon two-sided signed rank test was used for statistical comparison between pairs in subgroups II-IV for Vim and STN trajectories separately and P <.05 was considered significant. In order to approximate median trajectories for each patient group, data of all patients of each group were aligned to the target position (“ 0 "). In addition, standard deviation (s.d.) was calculated for all TLI trajectory positions. The lengths of subgroup II and III corresponding to $\mathrm{L}_{\mathrm{IC}}$ and $\mathrm{L}_{\mathrm{Cd} / \mathrm{Put}}$ as well as $\mathrm{L}_{\mathrm{Th}}$ when applicable were calculated in the median curves. TLI mean (m) and s.d. were determined and compared (Mann Whitney U-test) for subgroups II-IV in Vim and STN trajectories.

Ring and arc angles were noted for all patients and taken in consideration for the intra- and interpatient curve comparison. In order to do so and to be able to compare left and right arc angles, the left arc angles $\alpha$ were transformed by calculating $\alpha_{\text {trans }}=180^{\circ}-\alpha$.

\section{Comparison between microvascular blood flow and anatomy}

For quantitative comparison between anatomy and blood flow along the trajectories the number of hyperperfused sites was counted for the Vim and STN groups' respectively. As the blood flow in general is low (usually $<50$ a.u), 50 a.u was used as threshold to define a peak value together with the criteria as being at least twice as high as one of the surrounding microvascular blood flow measures. Using this principle the number of peaks were calculated for the following groups: A: 101-250, B: 251-500, and C > 500 a.u. Secondly the blood flow curves were inspected in order to remove overlapping between groups. The positions of the peaks were compared with TLI curves and the anatomy using the CT/MR images and 
Surgiplan ${ }^{\circledR}$. In 27 sites the TLI was less than 0.5 and consequently it was not possible to record the blood flow due to too little reflected light. If such peaks appeared next to high blood flow spots they were set to the maximum values 999.

The average blood flow ( $\mathrm{m} \pm$ s.d.) was calculated for II) white matter, III) the bridge between $\mathrm{Cd} / \mathrm{Put}$, or to the vicinity of ventricle, and IV) the target areas (including $-4 \mathrm{~mm}$ before the target down to the target). Wilcoxon two-sided signed rank test was used for statistical comparison between these subgroups in the Vim and the STN group separately and Mann Whitney U-test between Vim and STN for the three different subgroups. $\mathrm{P}<.05$ was considered significant.

\section{Results}

\section{TLI-trajectories}

Post-processing of the TLI signal showed that different typical anatomical regions could be identified in all patients. Examples of typical Vim and STN curves with marked anatomical regions and measures are presented in Fig. $3 \mathrm{c}-\mathrm{d}$. The median curves for Vim and STN respectively are presented in Fig. 4 together with the respective standard deviations. The average TLI for different regions is presented in Tab. 1 together with p-values. The TLI was significant higher in STN targets compared to Vim targets $(\mathrm{P}<.001)$.

\section{Vim-TLI-trajectories}

In the Vim recording the curve normally started with low values in the cortex (Fig. 2, 4). Individual fluctuations inside the cortex could be shown to be due to the presence of sulci. This was manifested as decreased TLI and increased perfusion. When the probe entered white matter the TLI increased and stayed approximately at this level until it crossed or passed in the vicinity of the $\mathrm{Cd} / \mathrm{Put} / \mathrm{Ventricle}$. The median length of $\mathrm{L}_{\mathrm{IC} 1}, \mathrm{~L}_{\mathrm{CD} / \mathrm{Put}}, \mathrm{L}_{\mathrm{IC} 2}$ and $\mathrm{L}_{\mathrm{Th}}$ were 31 , 4, 6 and $9 \mathrm{~mm}$ (Fig. 4). $d_{v}, 1_{v}$ varied between the patients. Mean values for $d_{v}$ and $l_{v}$ were $1.8 \pm$ $1.7 \mathrm{~mm}$ and $27.3 \pm 5.0 \mathrm{~mm}$ respectively. In one case, the probe went through the ventricle and this was seen as a more pronounced TLI-dip going almost to zero (Fig. 5a). In Vim patients, a significant statistical difference $(\mathrm{P}<.001)$ could be shown between white matter and $\mathrm{Cd} / \mathrm{Put}$ and between white matter and target area (Fig. 6). 
When comparing right and left implantation sides for each patient separately, often a shift or differences in dip properties were visible in the optical trajectories. An example of bilateral Vim curves is presented in Fig. 7a. Despite deviating dips in the curves, the last eight measurements follow each other. This agreement is also illustrated in the fluoroscopy image with overlapped implanted DBS electrodes. The ring angles were the same for both implantation sides and the frame was positioned in parallel with the AC-PC line. Another example with the same ring setting but an $11^{\circ}$ smaller left arc angle $\alpha_{\text {trans }}$ and a slight rotation of the frame to the right is illustrated in Fig. 7b. Along the AC-PC line the left target was more than $2 \mathrm{~mm}$ lateral and $2 \mathrm{~mm}$ anterior compared to the right side and the final position of the electrode tip was visible 3-4 mm below the planned target point. Those differences are probably the reason for the fact that the left side deviates from the expected pattern by not presenting a pronounced $\mathrm{Cd} / \mathrm{Put}$ dip while the right trajectory presents a typical TLI-Vim curve.

\section{STN-TLI-trajectories}

The STN curve started as well with low values in the cortex including some fluctuations caused by sulci and increased when entering white matter (Fig. 4, 5b). A dip was seen in patients when passing the bridge between Cd/Put. Depending on the direction of the trajectory this dip is more or less pronounced. The median length of $\mathrm{L}_{\mathrm{IC} 1}, \mathrm{~L}_{\mathrm{CD} / \mathrm{Put}}$ and $\mathrm{L}_{\mathrm{IC} 2}$ were 27, 5 and $17 \mathrm{~mm}$. Mean values for $\mathrm{d}_{\mathrm{v}}$ and $\mathrm{l}_{\mathrm{v}}$ were $5.9 \pm 3.9 \mathrm{~mm}$ and $25.3 \pm 6.2 \mathrm{~mm}$ respectively. The median and s.d. curves are presented in Fig.4. In STN patients, a significant statistical difference could be shown between white matter and $\mathrm{Cd} / \mathrm{Put} / \mathrm{Ventricle}(\mathrm{P}=.008)$ and between white matter and target area $(\mathrm{P}=.008)$ (Fig. 6). In one patient, measurements were done +4 $\mathrm{mm}$ below the STN target. This was manifested as decreasing TLI-values on both sides. Postoperative comparison with CT confirmed that the probe was very close to the substance nigra (Fig. 5b). As for Vim patients, curve shifts and changes in dip properties often existed in STN patients between right and left implantation side. In most cases they could be linked to changes in ring and arc angles.

\section{Microvascular blood flow}

Microvascular blood flow could be monitored at each step (Fig. 2, 5, 8, 9). High blood flow values were often seen in the cortex and close to sulci, and low blood flow values in white matter. In Vim patients, a slight increase was then again visible when entering the basal 
ganglia. The average blood flow for the different regions is presented in Tab.1 together with p-values. The blood flow was higher in Vim compared to STN targets $(\mathrm{P}=.01)$. No statistically significant difference existed in white matter and in $\mathrm{Cd} / \mathrm{Put}$ between $\mathrm{Vim}$ and STN. Furthermore, when considering STN and Vim trajectories separately, a statistically significant difference could be shown between white matter and $\mathrm{Cd} / \mathrm{Put}$ for $\operatorname{Vim}(\mathrm{P}=.005)$ trajectories and for white matter and target area for the $\operatorname{Vim}(\mathrm{P}=.001)$ and the $\mathrm{Cd} / \mathrm{Put}$ and target area for STN $(\mathrm{P}=.04)$.

285

In Tab. 2 a summary of the calculated high blood flow values and peaks are presented. There were three times higher blood flow spots along Vim trajectories compared to STN trajectories. Altogether 38 peaks (excluding a bleeding) were counted whereas 27 of them along Vim trajectories. Nine C-peaks (blood flow > 500 a.u.) were identified and they were all found in the cortical regions where sulci were present. Smaller i.e. A and B peaks were more common along Vim trajectories close to $\mathrm{Cd} / \mathrm{Put}$ and the ventricle. The heart rate calculated from the blood flow values agreed with the monitored rate during surgery.

In one Vim case a small bleeding was suspected during insertion of the probe and the measurement was terminated $5 \mathrm{~mm}$ from the precalculated target. This was seen as a deviation from the normal curve pattern (Fig. 8). Test stimulation after insertion of the DBSelectrode did not show any effect. Therefore the surgery was terminated and it appeared that the frame had been dislocated due to indeliberate head movement and thus the planned trajectory not followed. The suspected bleeding was confirmed with post-operative CT as diffuse and spread $<5 \mathrm{~mm}$ in diameter. A very small lesion $(<5 \mathrm{~mm})$ was visible on MRI on the same location 10 month later. The bleeding did not cause any permanent damage and the patient was then successfully re-operated. No measurements were done during the reoperation. Example of the median TLI and blood flow curves from $12 \mathrm{Vim}$ trajectories are presented together with the deviating curve caused by the bleeding is seen in Fig. 8 a-b. In Figure 9, corresponding median curves are presented for eight STN trajectories together with the deviation data due to the lesion in Vim. It is seen in Fig. 9a that the blood flow presents an A-peak close to the target region. 


\section{Discussion}

In the present study we have used the backscattered TLI signal as measured with a laser Doppler device in order to identify typical optical trajectories during DBS implantations. In addition the microvascular blood flow was recorded with mm-precision from cortex to the precalculated target areas. General TLI profiles were found for Vim and STN, however, with individual differences especially due to different trajectories, individual brain anatomy and the presence of vessels.

\section{Data acquisition}

This is the first study where both the microvascular blood flow and TLI signals recorded in the brain have been related to the respective anatomical position. This was possible due to construction of a mechanical device which allowed insertion with mm-precision all the way from cortex towards the Vim or the STN regions. Previous studies with reflectance spectroscopy and laser Doppler technique ${ }^{10-13}$ have used either fixed predetermined sites for the measurement or continuous insertion. It is not possible to make an exact correlation to the anatomy when insertion is done continuously. In addition, continuous insertion of the probe does not allow microvascular blood flow measurements as movement artifacts are superimposed on the laser Doppler signal. In this study, however the movement artifacts arising from the hand controlled screw (Fig. 2a) were used for identification of change in position and thus an important tool for relating the signal to the anatomical position.

The feed of commercially available microdrives are carried out in $\mu \mathrm{m}$-steps, either mechanically with a microscrew fixed to the ring, electrically via an electric motor or hydraulically with a piston pressure following the "master-slave-principal". These microdrive systems have a very high precision but they do not allow a recording with start in the cortex and all the way down to the deep brain structures. They are designed to be used with a limited movement range depending on the manufacturer and on the used frame. Usually the systems allow starting between 30 and $15 \mathrm{~mm}$ before the target and going up to $20 \mathrm{~mm}$ beyond, i.e. they have a total range between 35 and $50 \mathrm{~mm}$. As the aim of the present study was to obtain optical measurements along the whole trajectory in order to define typical optical trajectories, a homemade mechanical device with submillimetric precision was developed for the LSS. In the future, for routine use, when recordings might be concentrated on the deeper brain structures, the different microdrive systems can be used, after only minor modifications, with 
the optical probe. Future studies could also include TLI and blood flow measurements towards other target areas, e.g. the GPi, nucleus accumbens and the internal capsula. The technique is especially suited for targets where a clear and sharp border between grey and white matter is available. 3 T MRI and a smaller step size would increase the possibility to relate the optical measurements to anatomy even further. However, the step size always needs to be related to the maximum optical resolution achieved by the system and probe configuration.

In this study, recording of the TLI and blood flow was done for as short time as possible, but still not shorter than the time required for a clear and reliable signal. Previous investigations ${ }^{11}$ where the recording was set to $60 \mathrm{~s}$ showed that both the TLI and blood flow signals became stable as soon as the probe was fixed at the site. Therefore the measurement at each site was shortened considerably (to approximately $10 \mathrm{~s}$ ) and thus the total measurement time for each trajectory not longer than $15 \mathrm{~min}$. In clinical practice, when notes for scientific purpose are neglected, the measurement time can be reduced even more. The future system could as well incorporate a warning signal if the probe is deviating from the preplanned trajectory or if it is in the vicinity of a vessel structure. As the probe is forward looking i.e. the measured values appear before the site is passed, and the "look ahead" distance is in the mm-range ${ }^{18,19}$ it is possible to pause the insertion close to suspected vessel structures. Angular probes have also been suggested and used in relation to experimental RF-lesioning ${ }^{20}$ and clinical DBS implantations ${ }^{10}$. They have a feasibility to interrogate with the tissue perpendicularly from the probe shaft but have on the other hand a more complex design.

\section{Precision of anatomical data}

The limits of precision of anatomical atlases have often been discussed ${ }^{21-23}$ and when available anatomical structures should be identified directly on MR images. Nevertheless, in case of planning procedures on CT images, or in case of invisible or hardly identifiable structures even on MR images, atlases represent a helpful tool to relate the obtained measurements with the anatomical structures passed. During the anatomical comparison with the optical signals the Schaltenbrandt atlas was superimposed on the fused postoperative and preoperative images and the anatomical structures noted for axial, coronal and sagital directions. The fact that the structures identified on the three slice orientations at a specific position were not always identical could be due to a deviation of the atlas overlap of approximately 1-2 $\mathrm{mm}$. Furthermore there might be an error in the imaging itself related to 
the slice thickness, and to the reconstruction of the sagital and coronal images from the true axial ones. An additional error (approximately $1 \mathrm{~mm}$ ) could be induced during the identification of the final electrode position inside the electrode artifact ${ }^{17}$. Despite these different limitations in precision, it was possible to make a comparison between the trajectory and the optical signals from the target area towards the $\mathrm{Cd} / \mathrm{Put}$ region where the Schaltenbrandt atlas ends. Anatomical comparison for the cortex region and sulci had therefore to be done directly on the CT/MRI.

\section{Optical trajectories}

The optical trajectory differed between Vim and STN and there was a significant difference in grayness and blood flow between these targets, TLI was significantly lower and blood flow significantly higher in Vim compared to the STN. Previous studies using LDPM have also presented significant differences between STN and GPi in both grayness and blood flow ${ }^{11}$.

The STN curve was flatter, which is due to its longer passage through white matter. However, just a few millimeters deviation laterally or medial in the IC can result in small dips from structures in the thalamus or a double dip from the GPi/GPe and surrounding laminas. Clear double dips have previously been presented $^{10,13}$ during continuous spectroscopic optical measurements towards GPi. It is very likely that fractions of light from the same structures are picked up if the probe is passed more medial on the way to the STN. In two cases the probe was inserted $+4 \mathrm{~mm}$ below the planned target areas. In these cases, the following four measures were decreasing, and the probe tips found to be at the border to substance nigra. This sharp change in signal has also been presented by spectroscopic measurements ${ }^{10}$ and consequently an elevation in reflected light intensity appears to be a very significant marker 395 for STN targeting. It is not surprising that laser Doppler recordings and reflectance spectroscopy measurements present similar results. Previous studies have shown a very high correlation between these systems if data is analyzed at the same wavelength ${ }^{11}$.

The analysis of the average s.d. in Vim and STN trajectories (Figure 4) showed relatively high variations between patients in the first part of the optical trajectory. This can be explained by the presence of sulci in the cortex and by varying ring and arc angles. The variations at the beginning of the white matter region for STN and Vim patients are higher than in the second part of white matter. This is probably due to the fact that the position of the beginning of the white matter region is patient and angle dependent and in consequence some patients are already inside white matter while others are still in the cortex area. This explains 
as well why the average s.d. is lower in the second part of white matter in the median curve. The same explication is valid for the dip parts in Vim and STN median curve: the dips do not always appear exactly in the same distance to the target and their depth and width can depend on the trajectory angles or frame alignment to the AC-PC plane.

Variations when approaching the target area are less important as the target should be the same in all patients and as all patient data have been aligned to the target. One should be aware of the influence of brain individuality and different trajectory lengths which certainly influences the median trajectories. Due to the alignment to the target position, variations in patients will manifest with a higher probability further away from the target. Furthermore, small changes in the orientation of the trajectory would create longer distances between measurement points at the same level far from the target than close to it. This can explain as well an increasing variability with the distance from the target.

The reported intra- and interpatient variability in the $\mathrm{Cd} / \mathrm{Put} / \mathrm{Ventricle}$ dip region (see paragraph above and Figures 5 and 7) can explain the fact that no statistically significant differences could be revealed between this region and the Vim/ STN-TLI mean values: Even if in all patients a TLI decrease could be observed (Figure 6) when reaching the $\mathrm{Cd} / \mathrm{Put} /$ Ventricle, this decrease was more or less pronounced. In most patients the TLI values for $\mathrm{Cd} / \mathrm{Put} /$ Ventricle are lower than in the target area, but not in all of them. The exclusion for statistics of trajectories on which dips are very small (2 Vim and 3 STN trajectories) showed that target regions and $\mathrm{Cd} / \mathrm{Put} /$ Ventricle become significantly different $(\mathrm{p}<.05)$. In consequence the fact that no statistical significant difference could be shown when including all patients does not mean that the different regions cannot be identified when approaching the trajectory, but only that depending on the factors mentioned above these trajectories can differ. Nevertheless, a further study with decreased measurement steps is planned in order to establish more detailed relation between anatomy and TLI values on the last part of the trajectories towards Vim and STN.

In this study we have shown that high blood flow peaks are three times more common along Vim trajectories compared to STN trajectories. The high blood flow spots (A and B peaks) were often seen around the $\mathrm{Cd} / \mathrm{Put}$ and ventricle region in the Vim trajectory. C-peaks in the vicinity to sulci were common for both Vim and STN trajectories. High blood flow spots have also been found in a previous study when LDPM was used for stereotactic measurements at fixed predefined sites ${ }^{11}$. Here six out of 128 spots had high blood flow. At one of the spots a 
pronounced vasomotion pattern was recorded and a small vessel detected on the CT/MR. A tendency to vasomotion was seen in a few of the 38 high blood flow spots found in this study, but due to the relatively short measurement time at each site it was not possible to confirm this.

A small bleeding was discovered during insertion of the probe along one Vim-trajectory. Both the TLI and blood flow curves deviated from the expected shapes (Fig. 8). The TLI signal dropped drastically when it was expected to pass though white matter i.e. the internal capsula. At the actual bleeding neither the TLI nor the blood flow signal could be processed as there was too little light reflected back most probably due to coverage of blood on the fibers. A few $\mathrm{mm}$ further below, the signal recovered and presented high blood flow values related to A and B peaks $(\mathrm{n}=17,220 \pm 87$ a.u.). With knowledge of typical curve shapes for various DBS targets, both in respect to blood flow and TLI, it is possible to get an early warning sign related to vessels tracking and even bleedings during stereotactic insertion with this forward looking probe. Altogether we have used this type of optical probe for measurements and guidance for the DBS-electrode in 100 lead implantations ${ }^{24}$ and this is the only bleeding experienced. This indicates that the patient safety related to bleedings is increased over MER. More measurement series are however necessary to be performed in order to confirm this.

\section{Conclusion}

455 Laser Doppler is useful for intra-operative guidance during DBS implantation as simultaneous measurement of tissue grayness and microvascular blood flow can be done along the trajectory with mm-precision. Typical TLI-trajectories could be identified for Vim and STN based on which the passage through structures visible and invisible (such as the Vim) on the anatomical images can be confirmed. Further studies with higher resolution would increase the knowledge, especially about the target regions. Intraoperative laser Doppler perfusion monitoring measurements introduces the possibility for early bleeding prevention which is not possible with the other existing intraoperative techniques. 


\section{References}

1. Benabid AL. Deep brain stimulation for Parkinson's disease. Current Opinion in Neurobiology. 2003;13(6):696-706.

2. Benabid AL, Chabardes S, Seigneuret E, et al. Surgical therapy for Parkinson's disease. J Neural Transm Suppl. 2006(70):383-392.

3. Laitinen LV, Bergenheim AT, Hariz MI. Leksell's posteroventral pallidotomy in the treatment of Parkinson's disease. J Neurosurg. 1992;76(1):53-61.

470 4. Brundin P, Barker RA, Parmar M. Neural grafting in Parkinson's disease Problems and possibilities. Prog Brain Res. 2010;184:265-294.

5. Hemm S, Wårdell K. Stereotactic implantation of deep brain stimulation electrodes: a review of technical systems, methods and emerging tools. Med Biol Eng Comput. Jul 2010;48(7):611-624.

475 6. Laitinen L, Johansson GG, Sipponen P. Impedance and phase angle as a locating method in human stereotaxic surgery. J Neurosurg. Dec 1966;25(6):628-633.

7. Zrinzo L, Hariz M. Impedance recording in functional neurosurgery. In: Gildenberg PL, Lozano AM, Tasker R, eds. Textbook of stereotactic and functional neurosurgery 2008.

8. Gross RE, Krack P, Rodriguez-Oroz MC, Rezai AR, Benabid AL. 480 Electrophysiological mapping for the implantation of deep brain stimulators for Parkinson's disease and tremor. Mov Disord. Jun 29 2006;21(S14):S259-S283.

9. Giller CA, Johns M, Liu H. Use of an intracranial near-infrared probe for localization during stereotactic surgery for movement disorders. J Neurosurg. Sep 2000;93(3):498-505.

10. Giller CA, Liu H, German DC, Kashyap D, Dewey RB. A stereotactic near-infrared probe for localization during functional neurosurgical procedures: further experience. $J$ Neurosurg. Feb 2009;110(2):263-273.

11. Wårdell $\mathrm{K}$, Blomstedt $\mathrm{P}$, Richter $\mathrm{J}$, et al. Intracerebral microvascular measurements during deep brain stimulation implantation using laser Doppler perfusion monitoring. Stereotact Funct Neurosurg. 2007;85(6):279-286.

490 12. Antonsson J, Eriksson O, Blomstedt $\mathrm{P}$, et al. Diffuse reflectance spectroscopy measurements for tissue-type discrimination during deep brain stimulation. J Neural Eng. Jun 2008;5(2):185-190.

13. Johansson JD, Blomstedt $P$, Haj-Hosseini N, Bergenheim AT, Eriksson O, Wårdell K. Combined Diffuse Light Reflectance and Electrical Impedance Measurements as a Navigation Aid in Deep Brain Surgery. Stereotact Funct Neurosurg. Feb 18 2009;87(2):105113.

14. Jacobs P. Sterrad 100S, Sterilisation system. Johnson \& Johnson. 2006.

15. Nilsson GE, Salerud GE, Strömberg NOT, Wårdell K. Laser Doppler perfusion monitoring and imaging. In Biomedical Photonics Handbook. 2003:Chapter 15, 11-24. 
16. Wårdell K, Fors C, Antonsson J, Eriksson O. A laser Doppler system for intracerebral measurements during stereotactic neurosurgery. Conf Proc IEEE Eng Med Biol Soc. 2007;2007:4083-4086.

17. Hemm S, Coste J, Gabrillargues J, et al. Contact position analysis of deep brain stimulation electrodes on post-operative CT images. Acta Neurochir (Wien). Jul 505 2009;151(7):823-829; discussion 829.

18. Qian Z, Sunder S, Yeqing G, Giller C, Liu H. "Look-ahead distance" of a fiber probe used to assist neurosurgery: Phanton and Monte Carlo study. Optics express. 2003;11:18441855 .

19. Johansson JD, Fredriksson I, Wårdell K, Eriksson O. Simulation of reflected light intensity changes during navigation and radio-frequency lesioning in the brain. $J$ Biomed Opt. Jul-Aug 2009;14(4):044040.

20. Antonsson J, Eriksson $\mathrm{O}$, Lundberg $\mathrm{P}$, Wårdell K. Optical measurements during experimental stereotactic radiofrequency lesioning. Stereotact Funct Neurosurg. 2006;84(23):118-124.

515 21. Vayssiere N, Hemm S, Cif L, et al. Comparison of atlas- and magnetic resonance imaging-based stereotactic targeting of the globus pallidus internus in the performance of deep brain stimulation for treatment of dystonia. J Neurosurg. Apr 2002;96(4):673-679.

22. Schlaier J, Schoedel $\mathrm{P}$, Lange $\mathrm{M}$, et al. Reliability of atlas-derived coordinates in deep brain stimulation. Acta Neurochir(Wien). Nov 2005;147(11):1175-1180; discussion 1180.

520 23. Pinsker MO, Herzog J, Falk D, Volkmann J, Deuschl G, Mehdorn M. Accuracy and distortion of deep brain stimulation electrodes on postoperative MRI and CT. Zentralbl Neurochir. Aug 2008;69(3):144-147.

24. Wårdell K. Experience from optical measurements during 100 DBS implantations. XXth Congress of the European Society for Stereotactic and Functional Neurosurgery. Vol 90. Cascais/Lisbon, Portugal: Karger; 2012:8. 


\section{Legends to Figures}

530 Figure 1. a) Principle description of the measurement system. b) Leksell ${ }^{\circledR}$ Stereotactic System with the mechanical device and measurement probe. The probe tip is shown in the upper left corner.

Figure 2. Example of laser Doppler measurement using the mechanical device during stereotactic DBS implantation in Vim. a) Recording as presented in the operating room of: TLI (blue) and microvascular blood flow (yellow). b) Post-processing of optical trajectory and microvascular blood flow towards Vim. The values between the vertical dotted lines are presented in a). Values are presented in arbitrary units (a.u.). c) MRI with typical trajectory from cortex towards Vim.

Figure 3. Anatomical and typical normalised optical curves with marked distances used for analysis. a) Coronal cut $+5 \mathrm{~mm}$, b) Coronal cut $+2 \mathrm{~mm}$. c) Typical TLI-Vim curve d) Typical TLI-STN curve. Length of the section(s) passed in the internal capsule IC $\left(\mathrm{L}_{\mathrm{IC}}\right)$; the length of the passage through the bridge between cauduate nucleus $\mathrm{Cd}$ and Putamen (Put) ( $\left.\mathrm{L}_{\mathrm{CdPut}}\right)$, the distance from the border between the IC and the thalamus to the Vim-target $\left(\mathrm{L}_{\mathrm{Th}}\right)$.

Figure 4. Normalized median TLI for Vim $(n=12$, bleeding excluded) and STN ( $n=8$; lesion patient excluded) and the corresponding standard deviations. For length descriptions see Figure. 3.

Figure 5. a) Relative blood flow and TLI curves for Vim presenting measurement through the ventricle (left side) and the $\mathrm{Cd} / \mathrm{Put}$ (right side). High blood flow spots are seen in the cortex area in the vicinity to sulci (C-peaks) and in the vicinity to ventricles and Cd/Put (A and B peaks). b) Relative blood flow and TLI curves for STN. Measurements were done $+4 \mathrm{~mm}$ below the target i.e. to substance nigra (SN). For length descriptions see Figure 3.

Figure 6. Comparison between the three different anatomical regions in a) Vim and b) STN. Black bold curly brackets indicate significant difference between anatomical regions considering all patients.

555 Figure 7. Two examples of bilateral normalized TLI curves towards Vim with corresponding fluoroscopy are presented for a) identical and b) for $11^{\circ}$ different arc angles between right and left implantation sides. For length descriptions see Figure 3. 
Figure 8. Comparison between calculated median blood flow and TLI towards Vim and a patient recording including a bleeding. a) microvascular blood flow, b) normalized TLI.

560 Figure 9. Comparison between calculated median blood flow and TLI towards STN and a patient recording including a lesion. a) microvascular blood flow, b) normalized TLI. For length descriptions see Figure 3. 

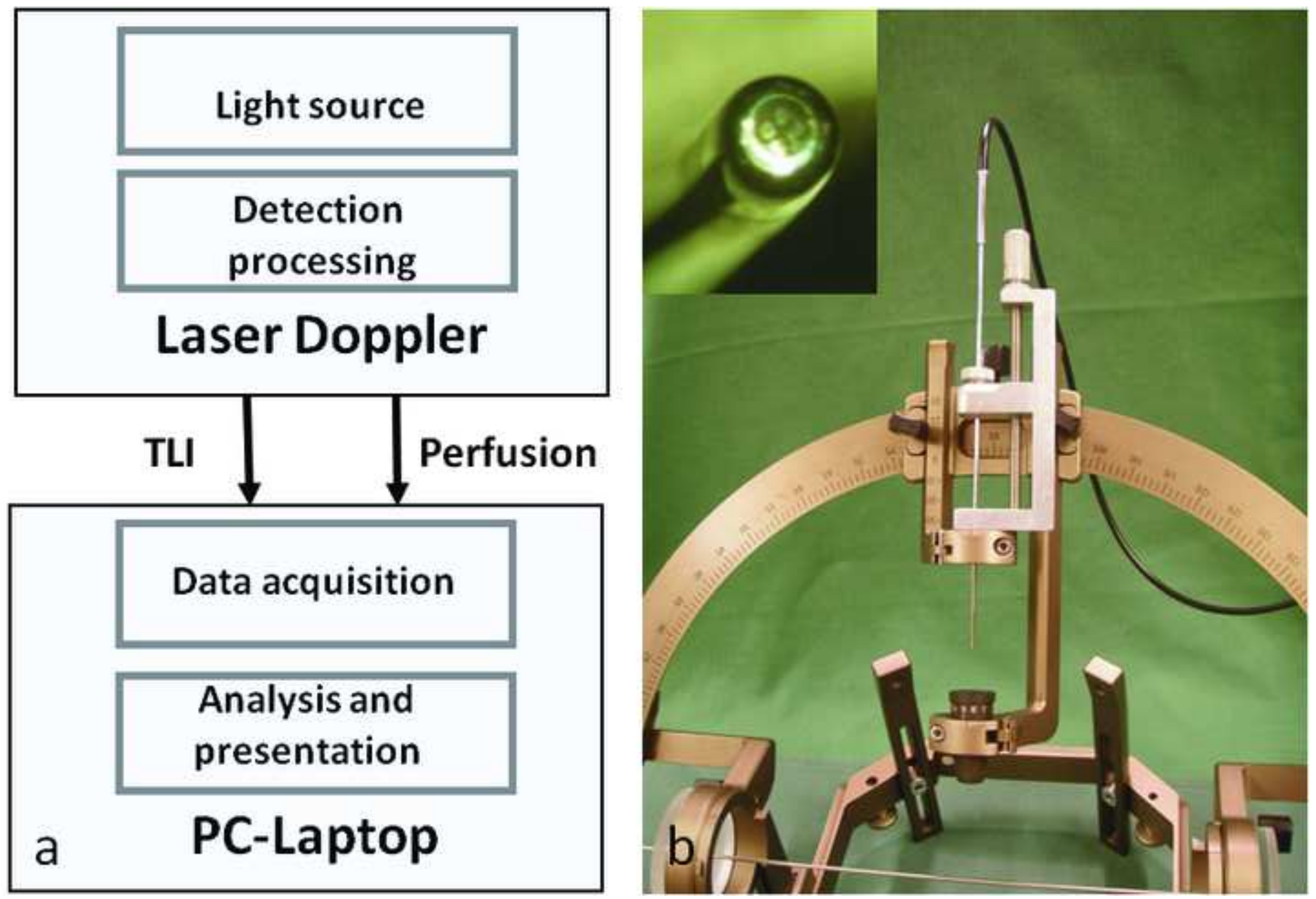

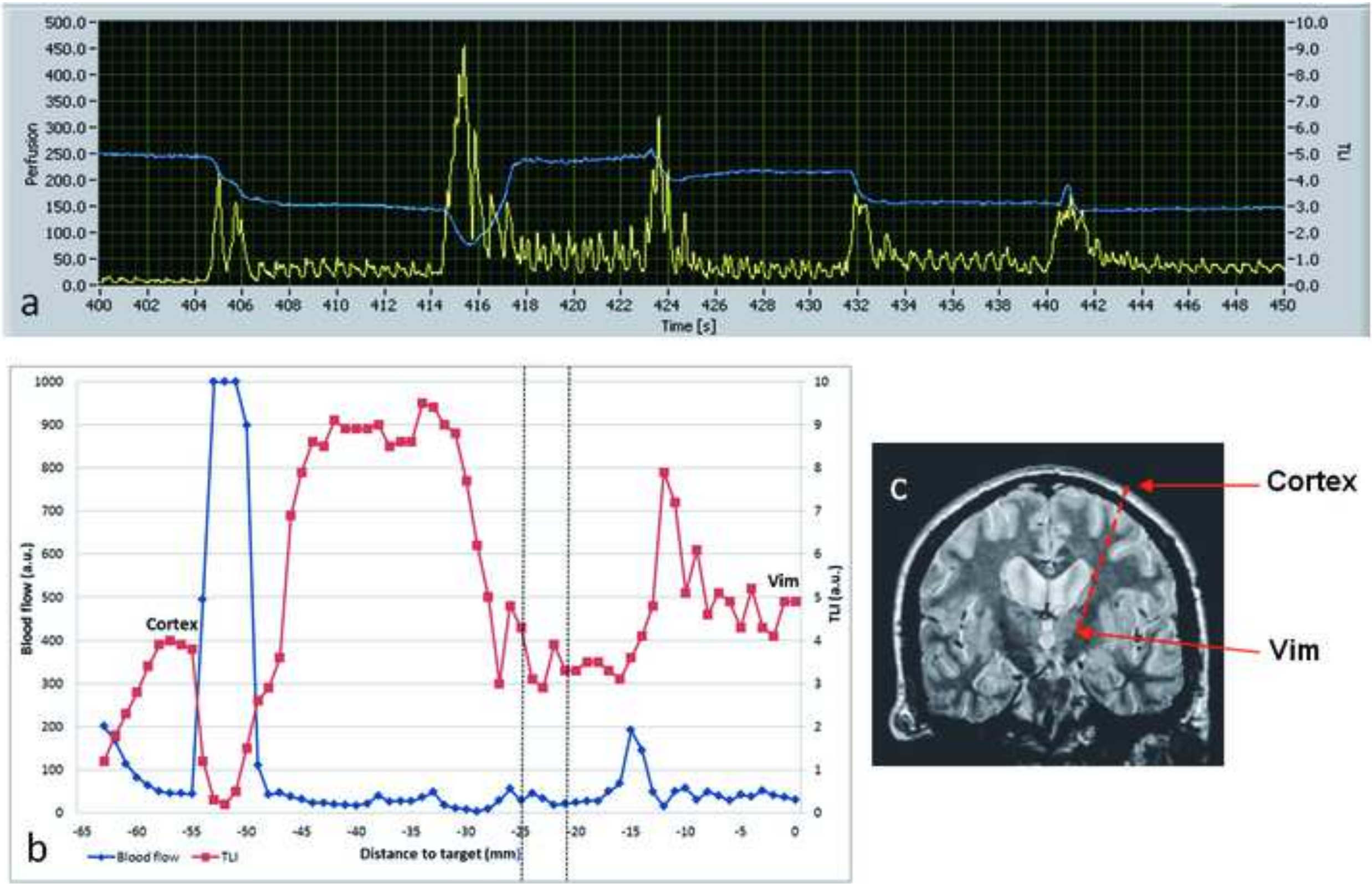

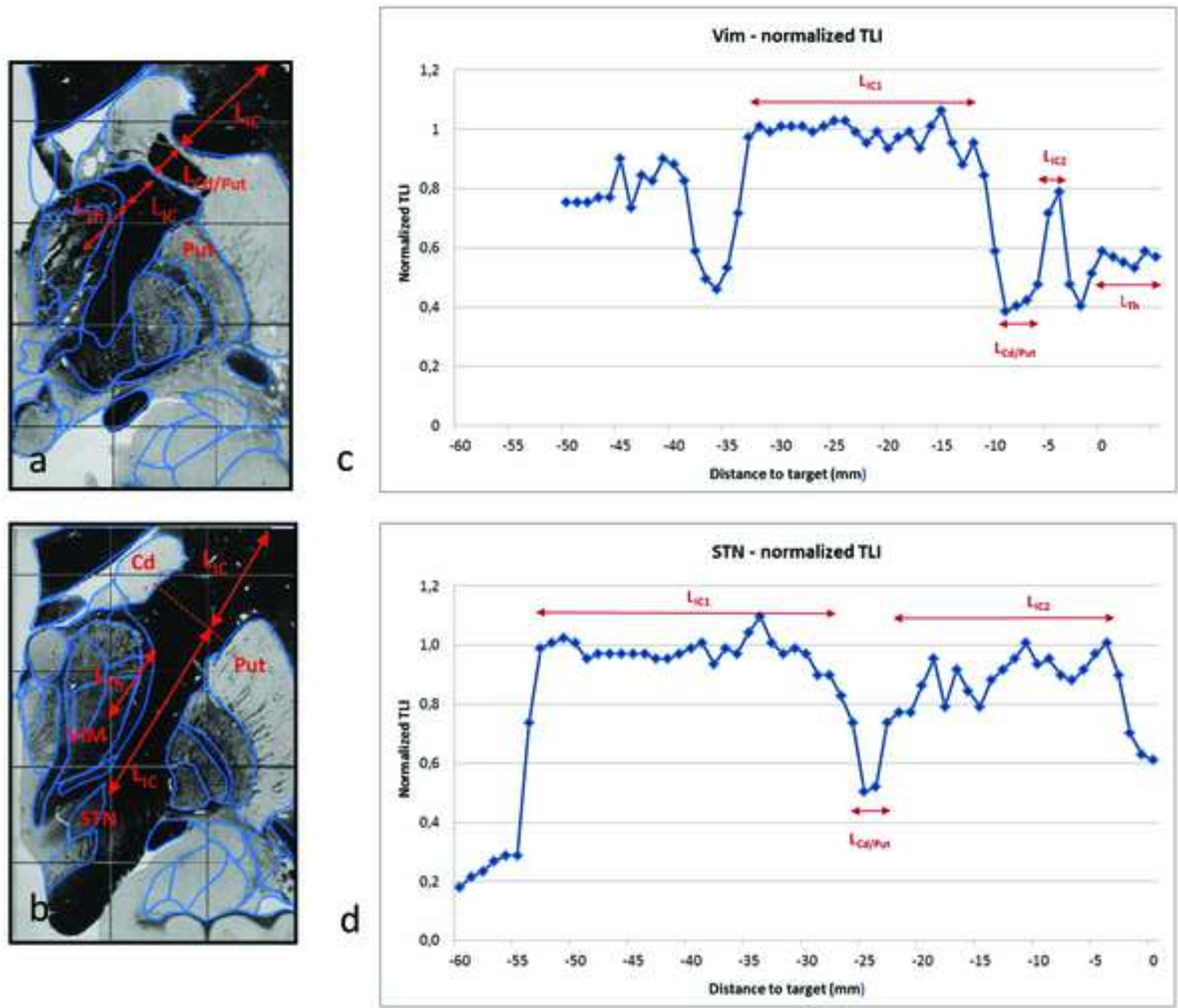


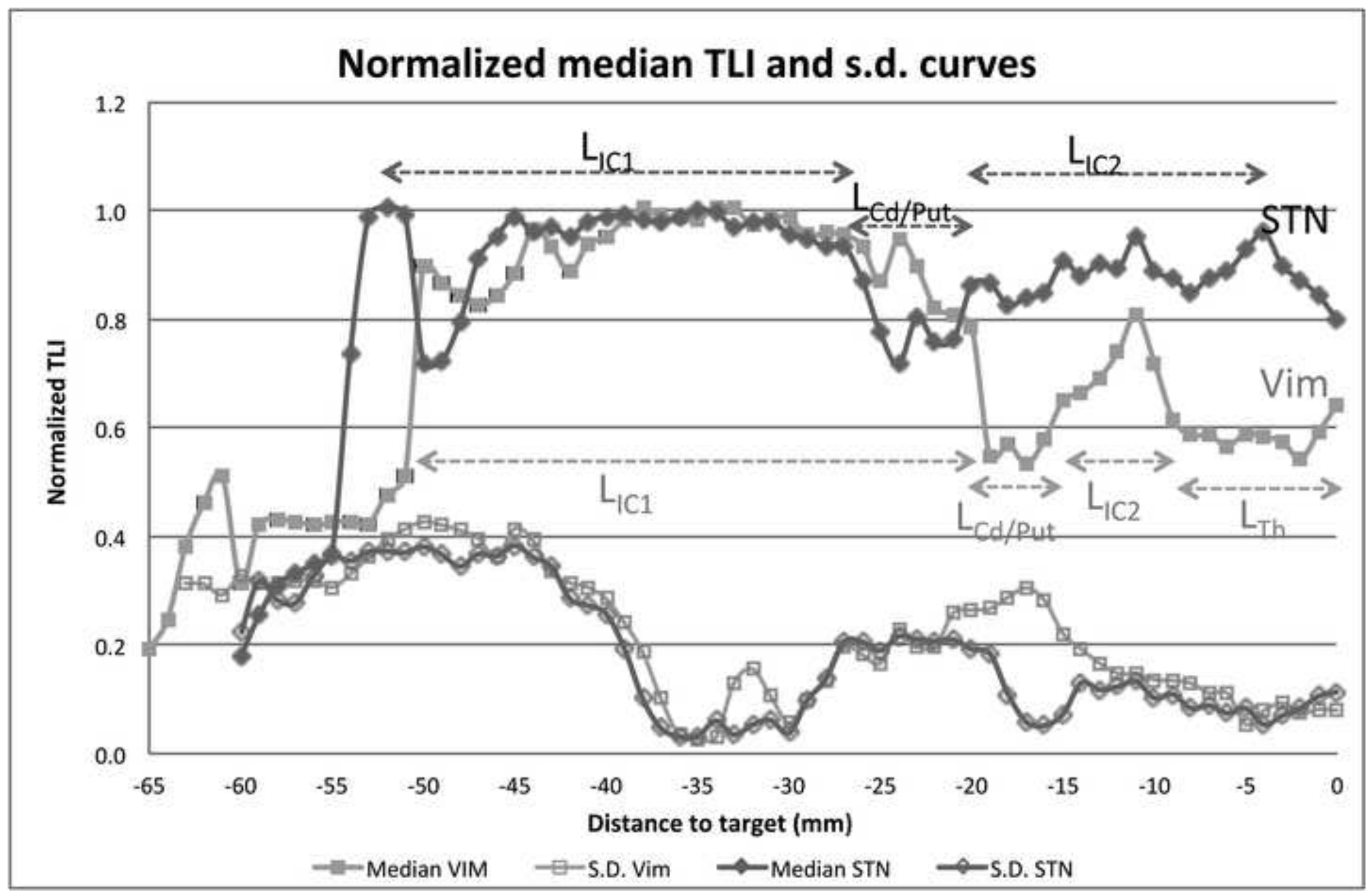


Figure 5

Click here to download high resolution image
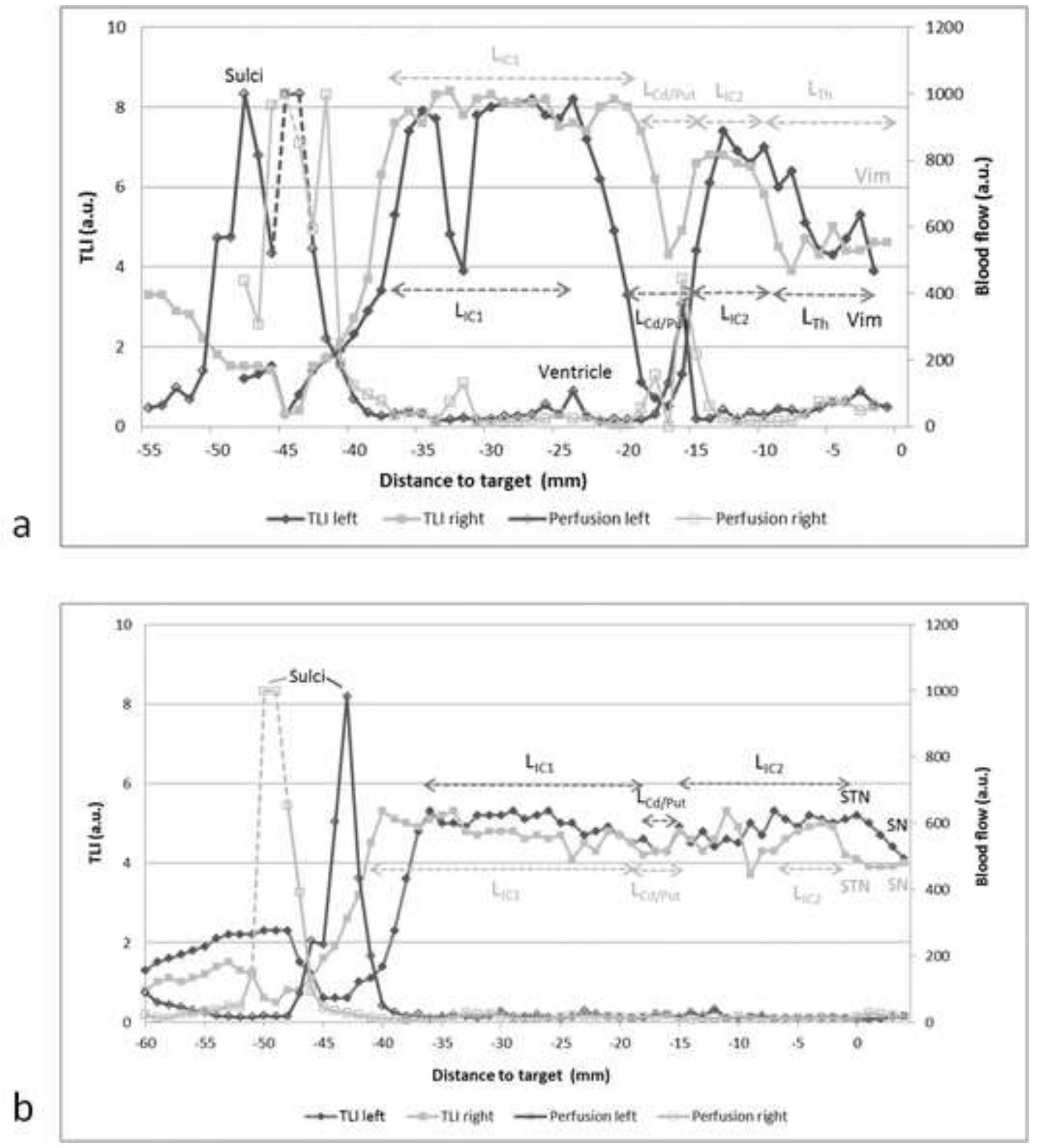

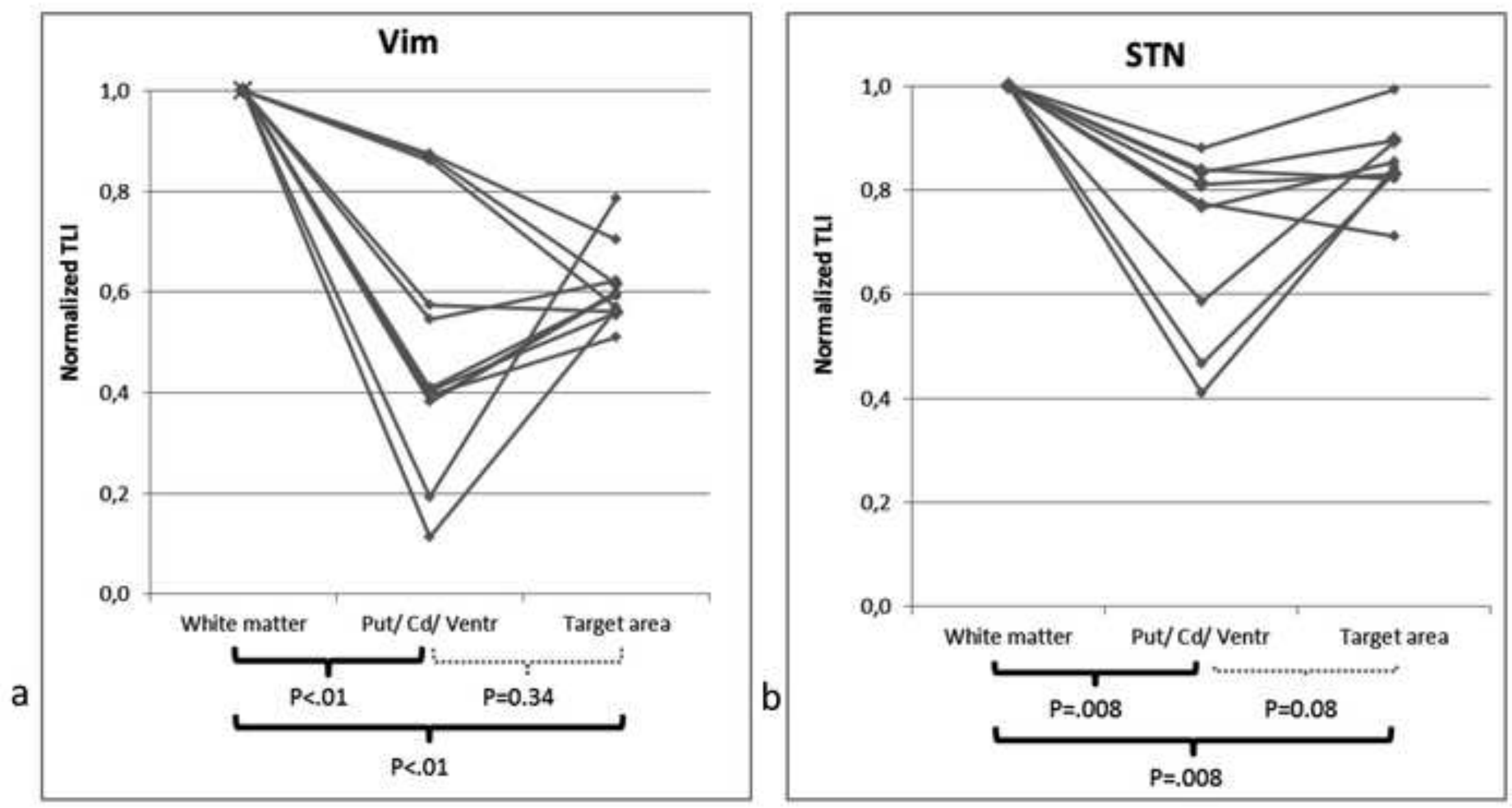

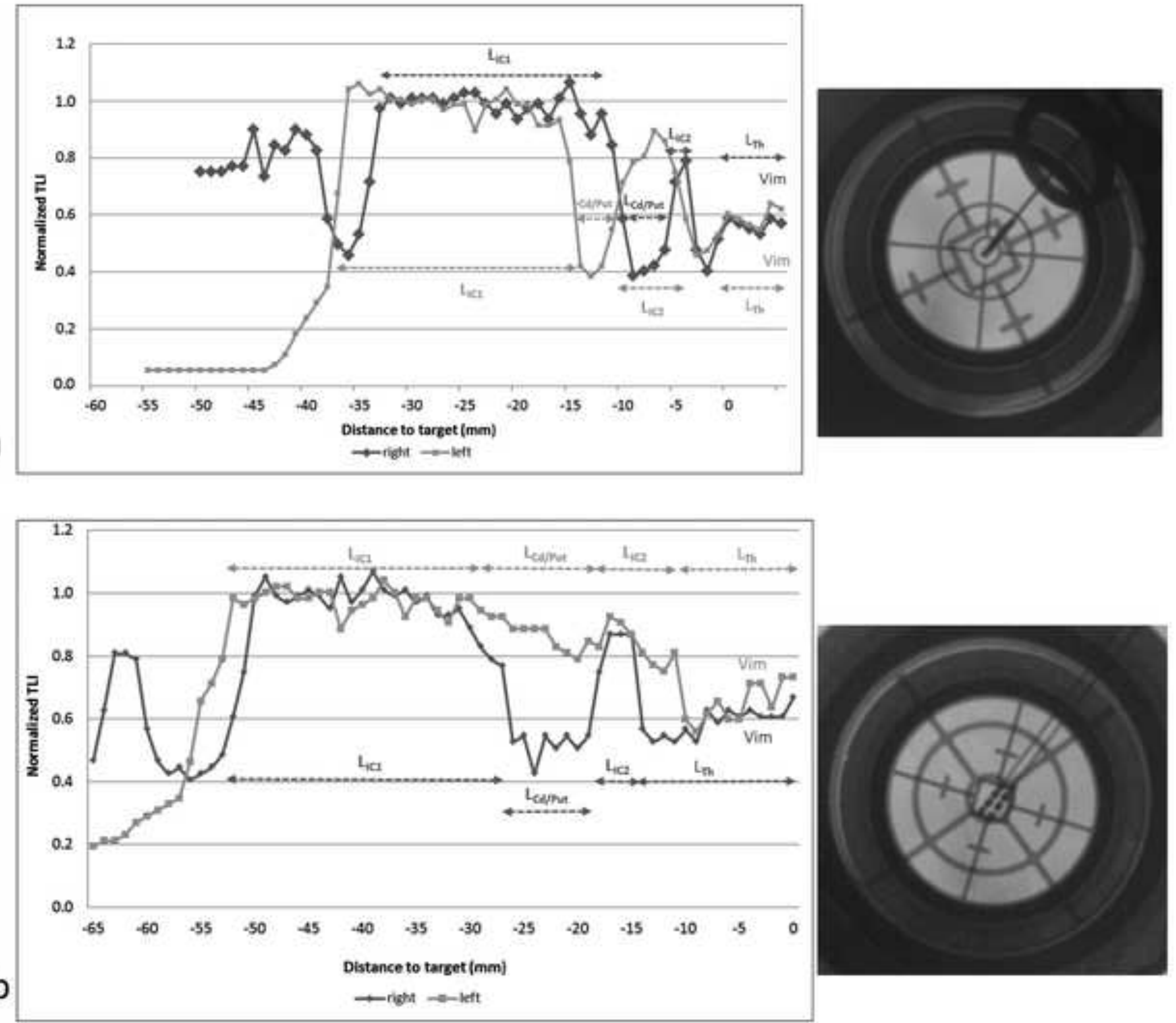

b

$$
\text { +right - o-kft }
$$



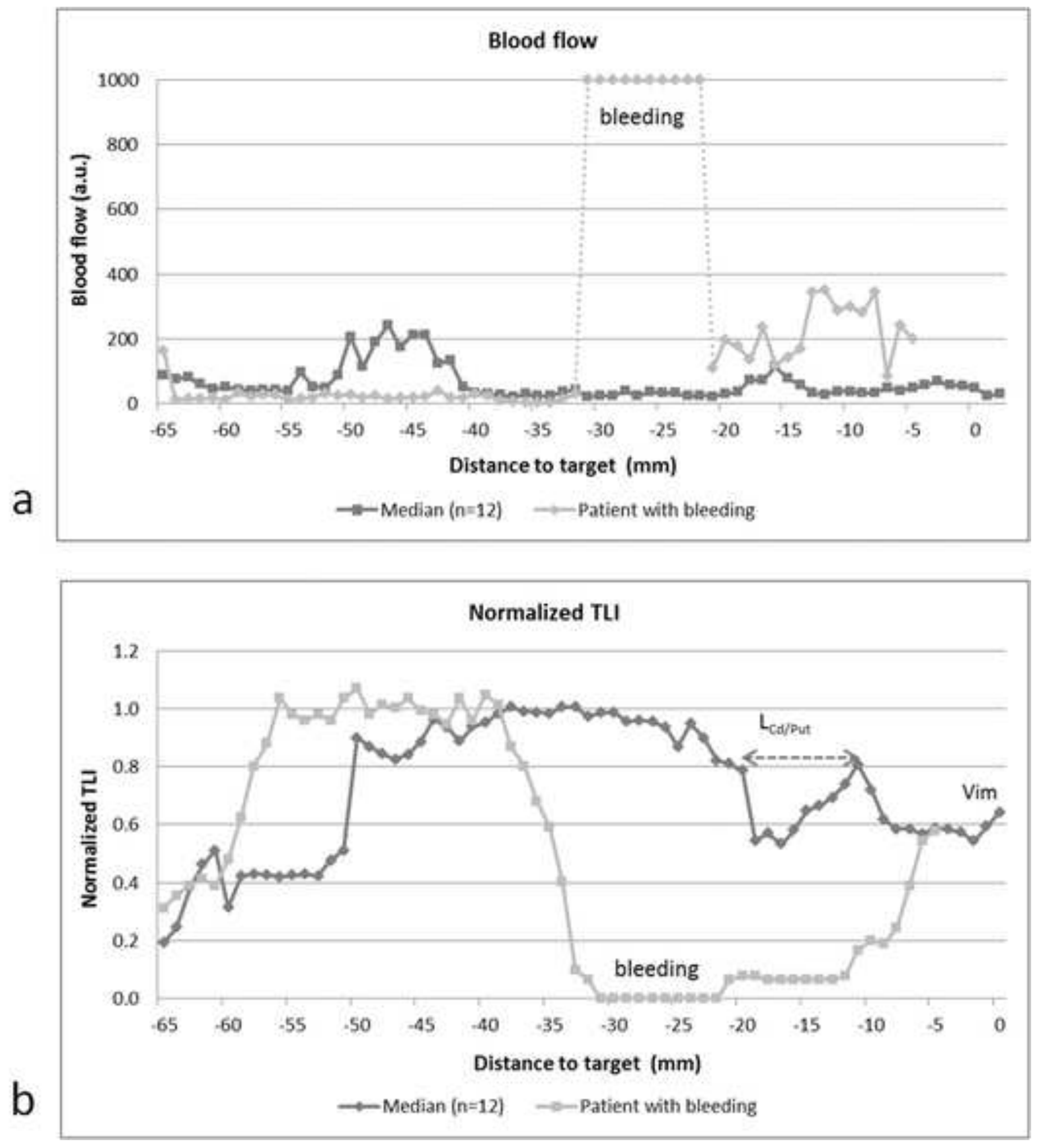
Figure 9

Click here to download high resolution image
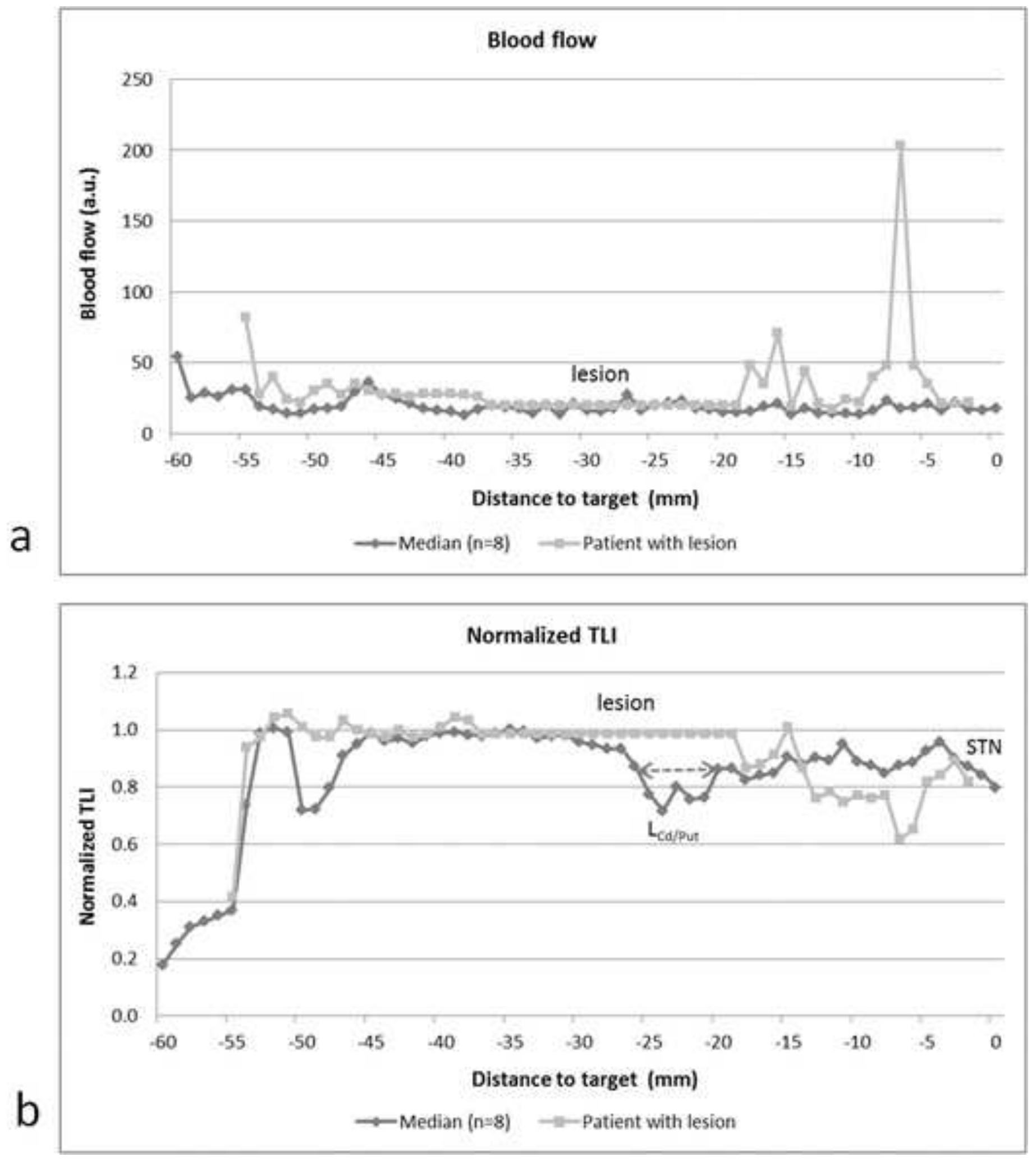
Tab. 1. Comparison of TLI and perfusion (mean \pm s.d.) between Vim and STN trajectories. *indicates a statistical significant difference.

\begin{tabular}{|c|c|c|c|c|}
\hline & & White & Cd/Put & Target \\
\hline TLI & Vim & 1 & $0.5 \pm 0.3$ & $0.6 \pm 0.1$ \\
\hline & STN & 1 & $0.7 \pm 0.2$ & $0.9 \pm 0.08$ \\
\hline & P-value & & 0.08 & $<.001^{*}$ \\
\hline Perfusion & Vim & $20.2 \pm 9.1$ & $93.8 \pm 82.9$ & $59.1 \pm 34.8$ \\
\hline & STN & $21.7 \pm 12.2$ & $40.1 \pm 34.4$ & $22.7 \pm 13.5$ \\
\hline & P-value & 0.51 & 0.15 & $<.01^{*}$ \\
\hline
\end{tabular}


Tab. 2. Summary of high perfusion values and peaks for VIM (bleeding excluded) and STN.

\begin{tabular}{|c|c|c|c|c|}
\hline $\begin{array}{c}\text { Perfusion } \\
\text { (a.u.) }\end{array}$ & $\begin{array}{c}\text { VIM } \\
\text { high values }\end{array}$ & $\begin{array}{c}\text { VIM } \\
\text { peaks }\end{array}$ & $\begin{array}{c}\text { STN } \\
\text { high values }\end{array}$ & $\begin{array}{c}\text { STN } \\
\text { peaks }\end{array}$ \\
\hline $101-250$ & 62 & 19 & 19 & 7 \\
\hline $251-500$ & 16 & 3 & 5 & 0 \\
\hline$>500$ & 40 & 5 & 8 & 4 \\
\hline Sum & $\mathbf{1 1 8}$ & $\mathbf{2 7}$ & $\mathbf{3 2}$ & $\mathbf{1 1}$ \\
\hline
\end{tabular}

